\title{
AVALIAÇÃO DO FOTOPERÍODO E DURAÇÃO DO PROTOCOLO COM PROGESTÁGENO SOBRE A CONCENTRAÇÃO PLASMÁTICA DE PROGESTERONA EM OVELHAS INSEMINADAS EM TEMPO FIXO (Evaluation of photoperiod and duration of the protocol with progestin on plasma progesterone concentration in ewes inseminated at fixed time)
}

\author{
João Lawrence Ortigosa, Helena Fabiana Reis de Almeida Saraiva, Marilice Zundt, \\ Rodrigo Mariuzzo Plens, Ines Cristina Giometti, Caliê Castilho
}

*Correspondência: calie@unoeste.br

RESUMO: Os objetivos do presente trabalho foram: avaliar, na primavera e verão (novembro a março), a concentração plasmática de progesterona em ovelhas lanadas e deslanadas criadas no oeste paulista; avaliar a concentração plasmática de progesterona em função do tempo de permanência do progestágeno (6, 9 ou 12 dias) em protocolo de inseminação artificial em tempo fixo (IATF). No experimento 1 foram utilizadas 12 ovelhas mestiças: 6 padrão Texel (Te) e 6 Santa Inês (SI), nas quais foram feitas, entre novembro e março, sete colheitas de sangue (C1 a $C 7)$, por venopunção da jugular, para posterior dosagem de progesterona $\left(\mathrm{P}_{4}\right)$ por radioimunoensaio (RIA). No experimento 2 foram utilizadas 38 ovelhas Te e SI divididas aleatoriamente em três grupos: G-6 ( $n=13)$; G-9 $(n=13)$; e G-12 ( $n=12)$. Inicialmente cada ovelha recebeu uma esponja intravaginal de progestágeno (D0) que permaneceu por 6 (G-6), 9 (G-9) ou 12 dias (G-12). Na retirada da esponja foram administrados, por via intramuscular, $0,1315 \mathrm{mg}$ de prostaglandina $F 2 \alpha$ (PGF2a) e 300 UI de gonadotrofina coriônica equina (eCG). A IATF, por via laparoscópica, foi feita a partir de 50 horas após a retirada do progestágeno. Trinta dias após foi realizado diagnóstico de gestação através de ultrassonografia transabdominal. Exp. 1. Com exceção da colheita 7 (C7), em todas as outras o grupo SI apresentou concentração de $\mathrm{P}_{4}$ estatisticamente superior $(\mathrm{P}<0,05)$ ao grupo Te. Exp. 2. No momento da retirada do progestágeno o G-12 apresentou concentração de $P_{4}$ significativamente $(P<0,05)$ menor $(0,342 \mathrm{ng} / \mathrm{mL})$ que o $\mathrm{G}-6(1,684 \mathrm{ng} / \mathrm{mL})$ e G-9 $(1,762 \mathrm{ng} / \mathrm{mL})$. No entanto não houve diferença na taxa de prenhez entre os grupos G-6 (76,9\%), G-9 (61,5\%) e G-12 (91,6\%). As ovelhas SI apresentaram concentração plasmática de $\mathrm{P}_{4}$ maior que as ovelhas Te nos períodos avaliados. $\mathrm{A}$ duração da permanência do progestágeno não afeta a taxa de prenhez em ovelhas.

Palavras-chave: ciclo estral; IATF; ovinos

ABSTRACT: The aims of this study were to evaluate, in spring and summer (November to March), the plasma progesterone concentration in woolly and hairless ewe sheep created in western São Paulo; evaluate the plasma progesterone concentration as a function of residence time of progestogen (6, 9 or 12 days) in TAI protocol (fixed-time artificial insemination). At experiment 1 we used 12 sheep cross breeds, 6 Texel (Te) and 6 Santa Inês (SI), in which were made between November and March, seven blood samples (C1 to $\mathrm{C} 7$ ), by jugular venipuncture for later determination of progesterone by radioimmunoassay (RIE). At experiment 2 we used 38 sheep cross breeds randomly divided into three groups: $G-6(n=13), G-9(n=13)$, and $G-12(n=12)$. Initially each sheep received an intravaginal sponge progestogen (D0) that has remained for 6 (G-6), 9 (G-9) or 12 days (G-12). At sponge removal were administered intramuscularly, $0.1315 \mathrm{mg}$ of PGF2a and $300 \mathrm{UI}$ eCG. The IATF, laparoscopic, was made from 50 hours after withdrawal of progestin. Thirty days after 
pregnancy diagnosis was performed by transabdominal ultrasound Exp 1. With the exception of crop 7 (C7), all other SI group $\mathrm{P}_{4}$ concentration showed statistically superior $(\mathrm{P}<0.05)$ than the group Te. Exp 2. Upon withdrawal of the progestogen $G-12 P_{4}$ concentration showed significantly $(P<0.05)$ lower $(0.342 \mathrm{ng} / \mathrm{mL})$ to G-6 $(1,684 \mathrm{ng} / \mathrm{ml})$ and G-9 $(1.762 \mathrm{ng} / \mathrm{mL})$. However there was no difference in pregnancy rates between groups G-6 (76.9\%), G-9 (61.5\%) and G-12 (91.6\%). The SI ewes showed plasma $\mathrm{P}_{4}$ greater than the Te sheep in periods. The duration of stay of progestin does not affect the pregnancy rate in ewes. The duration of stay of progestin does not affect the pregnancy rate in sheep.

Key Words: estrous cycle; FTAl; sheep 


\section{INTRODUÇÃO}

Para a expansão da ovinocultura no Brasil, torna-se necessário a busca de novas tecnologias que permitam ao produtor obter melhores resultados. Visando melhorar a produtividade do rebanho ovino, as investigações tem-se centrado no desenvolvimento de sistemas de manejo intensivo para atingir mais de um parto por ovelha a cada ano (Cameron et al., 2010). No entanto, a estacionalidade

reprodutiva, característica dessa espécie, é o principal fator a se levar em conta no momento da escolha do manejo reprodutivo a ser utilizado para atingir essa meta.

A ovelha pode apresentar-se como poliéstrica estacional ou contínua, dependendo do fotoperíodo da região, sendo a duração da estação reprodutiva definida primariamente pela latitude e secundariamente pela raça (Boland et al., 1990; Rosa \& Bryant, 2003; Sasa et al., 2011) . Portanto, se faz necessário conhecer o comportamento reprodutivo da raça a ser explorada, quer pela distinção de sua origem ou da região geográfica em que é criada, podendo assim requerer diferentes formas de manejo reprodutivo. Outro desafio também imprescindível é a viabilidade econômica para aumentar o uso dessas biotécnicas por parte dos produtores brasileiros. Portanto aprimorar e maximizar seus resultados, bem como diminuir seus custos são desafios que devem ser superados (Castilho et al., 2013).

O uso das técnicas de inseminação artificial (IA) aliada à sincronização de estro e da ovulação visando a inseminação artificial em tempo fixo (IATF) em animais geneticamente superiores permite acelerar o melhoramento genético do rebanho (Machado \& Simplício, 2001). Além disso, segundo Henderson et al. (1984), a sincronização do estro oferece ao produtor um grande número de vantagens práticas, tais como facilidade no manejo, o aproveitamento de pastagens, a otimização do macho e posterior comercialização de produtos da mesma idade.

Os métodos hormonais podem ser utilizados na estação reprodutiva ou na fase de anestro sazonal, variando apenas quanto ao protocolo a ser usado (Moraes et al., 2008). As tecnologias de sincronização do estro e ovulação em ovelhas são baseadas principalmente no controle da vida do corpo lúteo com prostaglandina $\mathrm{F} 2 \alpha$ ou utilizando tratamentos com progestágeno/progesterona associados à gonadotrofina coriônica eqüina (eCG) durante 12-14 dias (Gordon, 1997; Martemucci \& D'alessandro, 1999; Barrett et al., 2004). Entretanto, há estudos que apontam resultados semelhantes ou melhores de manifestação de estro e prenhez, tanto em ovelhas ciclando como em anestro, com a diminuição no tempo de permanência do progestágeno de 12 para 5 ou 6 dias (Viñoles et al., 2001; Knights et al., 2001; Martemucci \& D'alessandro, 2011). A justificativa para a diminuição do tempo de permanência do progestágeno, além do fator econômico, é embasada pelos trabalhos em bovinos que demonstraram que a alta permanência da progesterona induz um período maior de crescimento do folículo e conseqüente envelhecimento do oócito (Ginther et al., 1996).

Os objetivos do presente trabalho foram avaliar a concentração plasmática de progesterona no final da primavera e verão (novembro a março), com o intuito de conhecer a ciclicidade de ovelhas padrão Texel e Santa Inês, no oeste do estado de São Paulo; avaliar a concentração plasmática de progesterona em protocolo de IATF (inseminação artificial em tempo fixo) em função do tempo de permanência do progestágeno (6, 9 ou 12 dias). 


\section{MATERIAL E MÉTODOS}

O presente trabalho foi realizado na Universidade do Oeste Paulista UNOESTE, na cidade de Presidente Prudente, oeste do Estado de São Paulo (Latitude $22^{\circ} 07^{\prime} \mathrm{S}$ e Longitude $51^{\circ} 23^{\prime}$ ) no período de novembro de 2011 a março de 2012.

Experimento 1:

Foram realizadas, entre novembro de 2011 e março de 2012, sete colheitas de sangue (C1-7) por venopunção jugular de ovelhas padrão racial Texel $(T e ; n=6)$ e Santa Inês (SI; $n=6)$. Foram colhidas amostras de sangue em tubos de $15 \mathrm{~mL}$ heparinizados, mantidas em gelo por no máximo 1 hora pós-colheita, levadas ao laboratório Biotério II da UNOESTE (Universidade do Oeste Paulista, campus II, Presidente Prudente - SP) para serem centrifugadas a $750 \mathrm{~g}$ durante 15 minutos para a separação do plasma, o qual foi congelado a $-20^{\circ} \mathrm{C}$ para posterior dosagem de P4 pelo método de radioimunoensaio (RIA).

As colheitas foram feitas nos dias: 24/11/11 (C1), 01/12/11 (C2), 09/12/11 (C3), 21/12/11 (C4), 10/01/12 (C5), 02/02/12 (C6) e 08/03/12 (C7). A dosagem da concentração plasmática de P4 foi realizada no Laboratório de Endocrinologia Animal do curso de Medicina Veterinária da UNESP (Universidade Estadual Paulista, Campus de Araçatuba, SP) utilizando-se "kit" comercial (Coat-a-Count, Diagnostic Products Corporation, CA, USA). As dosagens de $\mathrm{P} 4$ foram realizadas em um ensaio cujo coeficiente de variação intraensaio do controle alto foi $10,02 \%$ e do controle baixo $0,31 \%$.

Experimento 2:

Foram utilizadas 38 ovelhas com padrão racial Texel (Te) e Santa Inês (SI), divididas de forma casualizada em três grupos de acordo a duração do tratamento com progestágeno: curto período (6 dias, G-6), médio (9 dias, G-9) ou longo (12 dias, G-12). Para manter proporção equilibrada de ovelha Te e SI dentro de cada grupo os mesmo ficaram da seguinte forma: Grupo 6 (G-6; $n=13$; $\mathrm{Te}=6$; $\mathrm{SI}=7$ ); Grupo 9 (G-9; $\mathrm{n}=13 ; \mathrm{Te}=$ 6; $\mathrm{SI}=7$ ); e Grupo 12 (G-12; $\mathrm{n}=12$; $\mathrm{Te}=$ $5 ; \mathrm{SI}=7$ ). As ovelhas apresentavam idade entre 4,0 e 6,0 anos, escore corporal médio 3,0 (escala 1 a 5), mantidas em pastagem de Panicum maximum cv. Mombaça, recebendo 250 g de concentrado com 18\% PB (proteína bruta), com acesso a água e sal mineral ad libitum.

A seguir foi iniciado protocolo hormonal para sincronizar as ovulações e permitir a IATF. Em estádio aleatório do ciclo estral (dia 0), cada ovelha recebeu uma esponja intravaginal contendo $0,33 \mathrm{~g}$ de progestágeno (acetato de medroxiprogesterona, Progespon $\AA$, Syntex, Brasil), permanecendo por 6 (G6), 9 (G-9) ou 12 dias (G-12). Na retirada das esponjas, todos os animais receberam $0,1315 \mathrm{mg}$ de prostaglandina F2 $\alpha$ (PGF - Sincrocio®, Ourofino, Brasil) e $300 \mathrm{UI}$ de gonadotrofina coriônica equina (eCG - Folligon $\square$, MSD, Brasil) ambos por via intramuscular (IM).

A IATF foi realizada depositando-se metade da dose da palheta de sêmen em cada corno uterino por via laparoscópica a partir de 50 horas após a retirada das esponjas. Foram utilizadas doses de sêmen congelado de reprodutor Dorper, com concentração espermática de 100×106 espermatozóides viáveis por dose.

Nos momentos da inserção do progestágeno (D0), retirada do progestágeno (R-P4), inseminação artificial (IATF) e 10 dias após a IATF (PIATF) foram colhidas amostras de sangue por venopunção jugular, em tubos de $15 \mathrm{~mL}$ heparinizados, mantidas em gelo por no máximo 1 hora póscolheita, levadas ao laboratório Biotério II da UNOESTE (Universidade do Oeste Paulista, campus II, Presidente Prudente - SP) para serem centrifugadas a $750 \mathrm{~g}$ durante 15 minutos para a separação do 
plasma, o qual foi congelado a $-20^{\circ} \mathrm{C}$. A dosagem da concentração plasmática de progesterona foi realizada no Laboratório de Endocrinologia Animal do curso de Medicina Veterinária da UNESP (Universidade Estadual Paulista, Campus de Araçatuba, SP) utilizando-se "kit" comercial (Coat-a-Count, Diagnostic Products Corporation, CA, USA), em um ensaio cujo coeficiente de variação intraensaio do controle alto foi de $2,54 \%$ e do controle baixo $0,09 \%$.

Trinta dias após a IATF foi realizado diagnóstico de gestação através da ultrassonografia transabdominal (Aloka SSD500, com probe convexa de 3,5 $\mathrm{MHz}$, Japão) e ao nascimento confirmouse o número de nascituros.

Análise Estatística

Para a comparação da concentração plasmática de $\mathrm{P} 4$ entre ovelhas lanadas e deslanadas, nas colheitas $\mathrm{C} 1$ a $\mathrm{C} 7$, foi utilizado o teste não paramétrico de Mann Whitney. Para a comparação da concentração plasmática de P4 entre os grupos G-6, G-9 e G-12, utilizou-se o teste de análise de variância em uma via (ANOVA one way) e as médias contrastadas pelo método de Tukey, e para as comparações em que a homogeneicidade de variâncias foi quebrado aplicou-se a correção de Welch com contrastes avaliados pelo método de Games-Howell. Para análise da taxa de prenhez entre grupos (G-6, G9 e G-12) recorreu-se ao teste de Quiquadrado de Pearson. Para todas as comparações foi considerada a associação significativa quando a estatística calculada foi correspondente a um $\square$ inferior a $5 \%(P<0,05)$. Todas as análises foram conduzidas com 0 software SPSS v. 13.0, conforme descrito por Field (2009).

\section{RESULTADOS E DISCUSSÃO}

\section{Experimento 1:}

As concentrações plasmáticas de $\mathrm{P} 4$ dos grupos de ovelhas Te e SI ao longo de novembro a março apresentaram diferenças entre os grupos raciais comparados (Tabela 1 e Figura 1). Com exceção da colheita 7 (C7), na quaL não foi observada diferença significativa $(P>0,05)$, em todas as outras o grupo de ovelhas SI apresentou concentração de P4 estatisticamente superior $(\mathrm{P}<0,05)$ ao grupo de ovelhas Te, devido a uma provável maior ciclicidade das ovelhas SI. Rodrigues et al. (2007), comparando esses grupos raciais criados em latitudes médias, como na região sudeste do Brasil, verificaram que as ovelhas SI apresentaram uma distribuição mais equilibrada de ciclos estrais ao longo do ano quando comparada com os animais Te, que apresentaram estacionalidade mais marcada, ciclando no período de outono e inverno e entrando na fase de anestro na primavera e no verão. Semelhante resultado pode ser observado no estudo de Sasa et al., (2002), que observou em ovelhas deslanadas criadas no estado de São Paulo maior regularidade de ciclos estrais durante o ano todo, ao passo que ovelhas lanadas estacionam sua ciclicidade a partir de agosto, na mesma região.

Tabela 1 - Média \pm desvio padrão (DP) da concentração plasmática de $\mathrm{P}_{4}$ (ng/mL) em ovelhas Texel (Te) e Santa Inês (SI) entre novembro de 2011 a março de 2012, no município de Presidente Prudente, oeste do estado de São Paulo. 

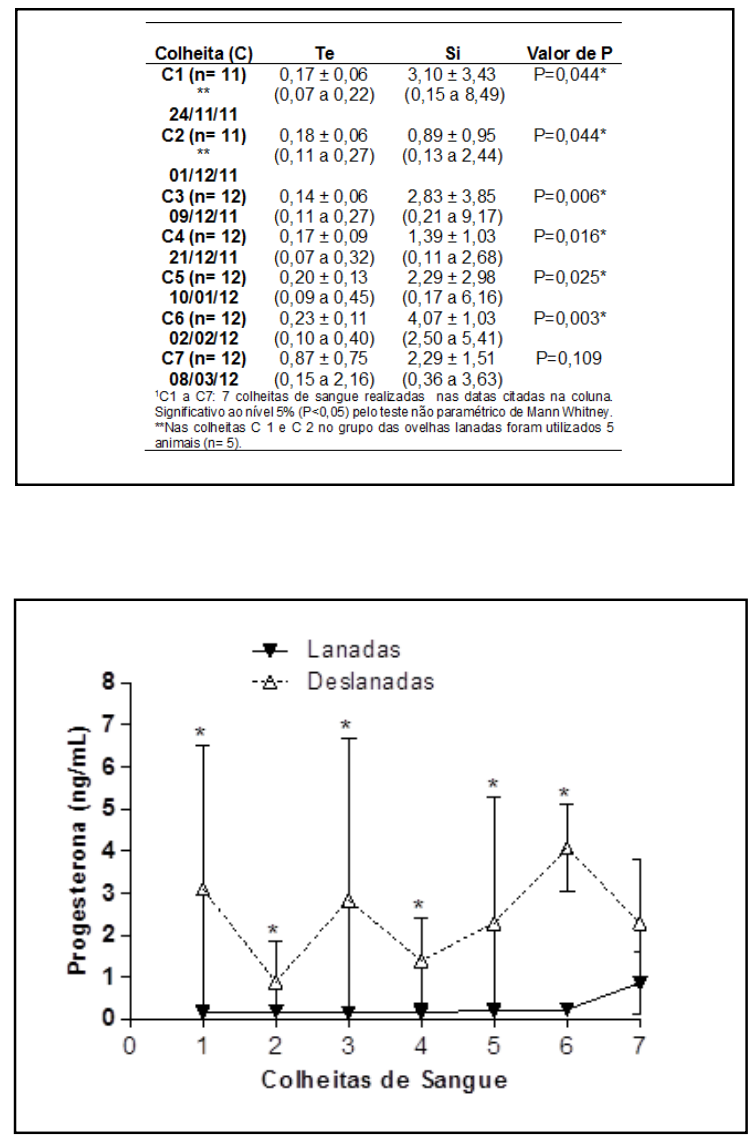

Figura 1 - Concentrações plasmáticas de progesterona $(\mathrm{ng} / \mathrm{mL})$ em ovelhas Texel e Santa Inês em oito colheitas, entre novembro de 2011 e março de 2012. $\left(^{*}\right)$ diferença significativa $(p<0,05)$ pelo teste não paramétrico de Mann Whitney.

\section{Experimento 2:}

As concentrações plasmáticas de

P4 em diferentes momentos, durante e após os protocolos hormonais para sincronizar a ovulação, estão demonstrados na Tabela 2. Não foi observada diferença significativa $(P>0,05)$ entre os grupos nos momentos de inserção do progestágeno (D0), no dia da IATF (IATF) e 10 dias após a IATF (P(ATF), mas no momento da retirada do progestágeno (R-P4) o G-12 apresentou concentração plasmática de P4 significativamente $\quad(P<0,05) \quad$ menor $(0,342 \mathrm{ng} / \mathrm{mL})$ que os grupos G-6 $(1,684$ $\mathrm{ng} / \mathrm{mL})$ e G-9 $(1,762 \mathrm{ng} / \mathrm{mL})$.

A P4 no G-12 no momento da retirada do progestágeno foi menor provavelmente pelo fato de que um maior tempo de permanência induz diminuição na P4 do CL endógeno. Progestágenos por 12 a 14 dias são amplamente utilizados na sincronização do estro em ovelha, apresentando, na maioria das vezes, mais de $90 \%$ de ovelhas em estro dentro de um período de 24 horas, e com uma taxa de concepção de 70 a $80 \%$ (Gordon, 1997; Evans et al., 2001). Esse 
método leva à diminuição sincrônica de progesterona (Roche et al., 1999).

Ao analisar a P4 por grupo, nos diferentes momentos, observou-se que a concentração no G-6 no dia da IATF $(0,22 \mathrm{ng} / \mathrm{mL})$ foi menor $(\mathrm{P}<0,05)$ que no D0 $(1,64 \mathrm{ng} / \mathrm{mL})$ e no dia da R-P4 $(1,76$ $\mathrm{ng} / \mathrm{mL})$, os quais não diferiram $(P>0,05)$ entre si (Tabela 1). Mas a concentração de P4 avaliada 10 dias após a IATF (4,9 $\mathrm{ng} / \mathrm{mL}$ ) foi significativamente maior $(P<0,05)$ que todos os momentos avaliados. As mesmas diferenças na concentração de $\mathrm{P} 4$, entre os momentos avaliados no G-6, foram observadas no grupo G-9.

Por outro lado, no G-12 a concentração plasmática de P4 não apresentou diferença $(P>0,05)$ entre D0 (2,65 ng/mL) e R-P4 (0,342 ng/mL), mas a P4 no dia D0 foi significativamente maior $(P<0,05)$ que no dia da IATF $(0,198$ $\mathrm{ng} / \mathrm{mL}$ ). Neste grupo a concentração de P4 10 dias após a IATF $(6,916 \mathrm{ng} / \mathrm{mL})$ foi superior $(P<0,05)$ aos momentos $\mathrm{R}-\mathrm{P} 4 \mathrm{e}$
IATF, mas estatisticamente semelhante ao D0 $(P>0,05)$.

Em todas as fêmeas estudadas houve queda da P4 abaixo de $2 \mathrm{ng} / \mathrm{mL}$ após a aplicação de PGF, dado observado na colheita de sangue no momento da IATF (50h após aplicação de PGF). O estro não ocorre, até que os níveis de P4 declinarem a cerca de 1 $\mathrm{ng} / \mathrm{mL}$ de plasma (Stabenfeldt et al., 1969; Johnson et al., 1996).

Tabela 2 - Concentrações plasmáticas de $P_{4}(n g / m L)$ em grupos de ovelhas com diferentes tempos de inserção de implante (6, 9 ou 12 dias), nos momentos inserção do progestágeno (D0), retirada do progestágeno (R-P4), dia da IA (IATF) e 10 dias pós IATF (PIATF), em protocolo de sincronização da ovulação. 


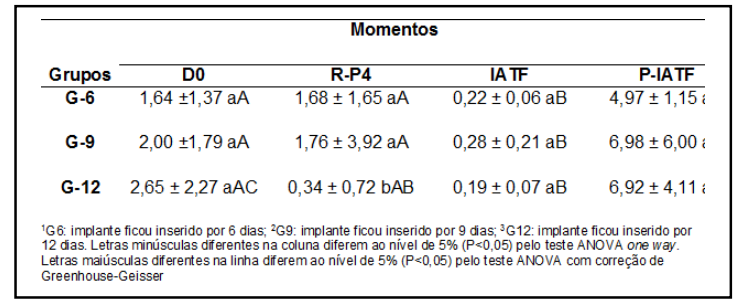

No presente estudo, devido ao pequeno número experimental de animais, não foi possível detectar diferença estatisticamente significativa $(P>0,05)$ na porcentagem de prenhez entre os grupos avaliados (Tabela 3).

Tabela 3 - Porcentagem (\%) e número de prenhez em ovelhas nos grupos com curta (G-6), média (G-9) e longa (G-12) permanência do progestágeno em protocolo hormonal para IATF por laparoscopia com sêmen congelado.

\begin{tabular}{|c|c|c|c|}
\hline $\begin{array}{l}\% \\
\text { Prenhez } \\
\text { Letras iguais na }\end{array}$ & $\begin{array}{c}\text { G-6 } \\
76,9 a \\
(10 / 13) \\
m(P>0,05)\end{array}$ & $\begin{array}{c}\text { G-9 } \\
61,5 a \\
(8 / 13) \\
\text { do qui-quad }\end{array}$ & $\begin{array}{c}\text { G-12 } \\
91,6 a \\
(11 / 12) \\
\text { arson. }\end{array}$ \\
\hline
\end{tabular}

Porém, o tratamento de curta duração com progestágeno (G-6) resultou em $76,9 \%$ de prenhez, a qual foi numericamente $10,4 \%$ maior que no tratamento de média duração (G-9, $61,53 \%$ ). No grupo de longa duração (G12) a porcentagem de prenhez $(91,6 \%)$ foi $14,7 \%$ e $30,1 \%$, maiores que nos grupos G-6 e G-9, respectivamente. Entretanto, estatisticamente, a diminuição no tempo de uso do progestágeno de 12 para 6 dias não alterou os resultados das taxas de prenhez.

Estudo de Flynn et al. (2000) demonstrou que um tratamento por 14 dias com P4, na ausência do $C L$, não teve eficácia em manter um feedback negativo na frequência dos pulsos de LH ao longo do tempo, levando a um crescimento prolongado do folículo ovulatório. Existem relatos de que a administração de P4 em vacas por longos períodos promove um baixo turnover de folículos durante o ciclo estral, resultando na ovulação de folículos persistentes e prejudicando as taxas de prenhez, ao passo que o tratamento com períodos mais curtos de P4 permite a ovulação de folículos jovens e consequentemente taxas de prenhez mais atrativas (Viñoles et al., 2001; Bartlewski et al., 2011). Esse fato não foi observado no presente estudo, onde a maior taxa de prenhez $(91,6 \%)$, embora não significativa, foi observado no grupo de maior duração G-12. Em outro trabalho, Özyurtlu et al. (2011) obtiveram semelhantes taxas de estro, prenhez, parição, fecundidade e duração do estro comparando protocolos de curta (7 dias) e longa duração (14 dias). Taxas semelhantes de prenhez puderam ser verificadas por Ustuner et al. (2007) em tratamentos de 6 e 12 dias, concluindose que protocolos de curta duração são tão eficientes quanto os de duração mais longa, principalmente se utiliza a PGF como agente luteolítico, potencializando a sincronização do estro e da ovulação. $\mathrm{Na}$ estação reprodutiva, o protocolo de 12 dias de P4 com aplicação de eCG e PGF também pode ser substituído por um protocolo mais curto de 7 dias de P4 sem prejuízos nos índices de sincronização de estro e fertilidade, podendo haver aumento da taxa de partos múltiplos ao se associar o $\mathrm{GnRH}$ a este protocolo de curta duração (Karaca et al., 2009).

\section{CONCLUSÃO}

As ovelhas Santa Inês apresentaram concentração plasmática de P4 maior que as ovelhas Texel nos períodos avaliados, demonstrando que as ovelhas Texel exibem estacionalidade reprodutiva nessa região.

A duração da permanência do progestágeno não afeta a taxa de prenhez em ovelhas, entretanto, as 
ovelhas tratadas com progestágeno por 12 dias apresentaram, na retirada do implante, menor taxa de P4, e maior taxa numérica de prenhez, quando comparadas com as ovelhas dos tratamentos 9 e 6 dias.

\section{REFERÊNCIAS}

BARRET, D. M. W.; BARTLEWSKI, P. M.; BATISTA-ARTEAGA, $M$. et al. Ultrasound and endocrine evaluation of the ovarian response to a single dose de $500 \mathrm{UI}$ of eCG following a 12 days treatment with progestogen-releasing intravaginal sponges in the breeding and non breeding seasons in ewes. Theriogenology, v.61, n.2, p. $311-327, \quad 2004$. DOI: 10.1016/S0093691X(03)00215-2.

BARTLEWSKI, P. M.; BABY, T. E.; GIFFIN, J. L. Reproductive cycles in sheep. Anim. Reprod. Sci., v. 124, n. 3-4, p. 259-268, 2011. DOI: 10.1016/j.anireprosci.2011.02.024.

BOLAND, M. P.; CROSBY, F.; O CALLACHAN, $D$. Artificial control of the breeding season in ewes. Irish Veterinary Jounal, v. 43, p. 2-6, 1990.

CASTILHO, C.; ALMEIDA, M. F.; COSTA, M. Z. et al. Protocolos de indução e sincronização do estro em ovelhas. Ciênc. anim. bras., v.14, n.1, p.91-97, 2013. DOI: 10.5216/cab.v14i1.13378.

EVANS, A. C.; FLYNN, J. D.; QUINN, K. M. et al. Ovulation of aged follicles does not affect embryo quality or fertility after a 14-day progestagen estrus synchronization protocol in ewes. Theriogenology, n.56, v.5, p.923-936, 2001. Disponível em: <http://www.ncbi.nlm.nih.gov/pubmed/11665893

FIELD, A. Descobrindo a estatística usando o SPSS. 2 ed. Porto Alegre: Artmed, 2009.

FLYNN, J. D.; DUFFY, P.; BOLAND, M. P. et al. Progestagen synchronization in the absence of a corpus luteum results in the ovulation of a persistent follicle in cyclic ewe lambs. Anim. Reprod. Sci. v.62, n.4, p.285-296, 2000. Disponível em: <http://www.animalreproductionscience.com/arti cle/S0378-4320(00)00124-X/abstract>

GINTHER, O. J.; WILTBANK, M. C.; FRICKE, P. $M$. et al. Selection of the dominant follicle in cattle. Biol. Reprod, v. 55, n.6, p. 1187-1194, 1996. DOI: 10.1095/biolreprod55.6.1187.

GORDON, I. Controlled Reproduction in Sheep and Goats. CAB International: New York, 1997. 450p.
HENDERSON, D. C. ; DOWNING, J. M.; BECK, N. F. G. et al. Oestrus synchronization in ewes: a comparison of prostaglandin $\mathrm{F} 2 \square$ than salt with a progestagen pessary. Anim. Prod., v. 39, n.2, p. 229-233, $1984 . \quad$ DOI: $10.1017 / S 0003356100041854$.

JOHNSON, S. K.; DAILEY, R. A.; INSKEEP, E. K. et al. Effect of peripheral concentrations of progesterone on follicular growth and fertility in ewes. Domest. Anim. Endocrinol. v. 13, n. 1, p. 69-79, 1996. Disponível em: $<$ http://www.domesticanimalendo.com/article/07 39-7240(95)00045-3/abstract>

KARACA, F.; ATAMAN, M. B.; COYAN, K. Synchronization of estrus with short- and longterm progestagen treatments and the use of $\mathrm{GnRH}$ prior to short-term progestagen treatment in ewes. Small Rumin. Research. v.81, n.2-3, p.185-188, 2009 . DOI: 10.1016/j.smallrumres.2008.12.002.

KNIGHTS, M.; HOEHN, T.; LEWIS, P. E. et al. Effectiveness of intravaginal progesterone inserts and $\mathrm{FSH}$ for inducing synchronized estrus and increasing lambing rate in anestrous ewe. J. Anim. Sci., v. 79, n.5, p. 1120-1131, 2001. Disponível em: <http://www.journalofanimalscience.org/content/ 79/5/1120.long>

MACHADO, R.; SIMPLíCIO, A. A. Avaliação de programas hormonais para a indução e sincronização do estro em caprinos. Pesq. Agropec. Bras. v. 36, n. 1, p. 171-178, 2001. Disponível <http://www.scielo.br/pdf/pab/v36n1/4239.pdf>

MARTEMUCCI, G.; D'ALESSANDRO, A. G. Synchronization of oestrus and ovulation by short-time combined FGA, PGF2 $\alpha$, GnRH, eCG treatments for natural service or Al fixed-time. Anim. Reprod. Sci., v. 123, n. 1-2, p. 32-39, 2010. DOI: 10.1016/j.anireprosci.2010.11.007.

MORAES, J. C. F.; SOUZA, C. J. H.; GONÇALVES, P. B. D. et al. Controle do estro e da ovulação em ruminantes. In: GONÇALVES, P. B. D.; FIGUEIREDO, J. R.; FREITAS, V. J. F. Biotécnicas aplicadas à reprodução animal. 2. ed. São Paulo: Roca, 2008, cap. 3, p. 33-56.

ÖZYURTLU, N.; SERHAN, A. Y, S.; KUÇUKASLAN, I. et al. Effect of subsequent two short-term, short-term, and long-term progestagen treatments on fertility of Awassi ewes out of the breeding season. Ank. Üniv. Vet. Fak., v.58, p.105-109, 2011. Disponível em: $<$ http://dergiler.ankara.edu.tr/dergiler/11/1529/16 797.pdf> 
RODRIGUES, P. A.; COELHO, L. A.; NONAKA, K. O. et al. Annual characteristics of estrous activity in wool and hair ewe lambs under subtropical condictions. Sci. Agric., v.64, n.5, p.468-475, 2007. DOI: 10.1590/S010390162007000500003.

ROCHE, J. F.; AUSTIN, E. J.; RYAN, M. et al. Regulation of follicle waves to maximize fertility in cattle. J. Reprod. Fertil. Suppl. v. 54, p. 61-71, $1999 . \quad$ Disponível em: <http://www.ncbi.nlm.nih.gov/pubmed/10692845 $>$

ROSA, H. J. D.; BRYANT, M. J. Seasonality of Reproduction in Sheep. Small Ruminant Research, v.48, N.3, p.155-171, 2003. DOI: 10.1016/S0921-4488(03)00038-5.

SANTOS, G. M. G.; SANTOS, S. K. C.; STERZA, F. A. M. et al. Desempenho reprodutivo de ovelhas mestiças lanadas e deslanadas submetidas a protocolo hormonal a base de progestágeno e eCG, durante a contraestação reprodutiva. Semina: Ciências Agrárias. v. 32, n. 2, p. 723-732, 2011. DOI: 10.5433/16790359.2011v32n2p723.

SASA, A.; TESTON, D. C.; RODRIGUES, P. A. et al. Concentrações Plasmáticas de Progesterona em Borregas Lanadas e Deslanadas no Período de Abril a Novembro, no Estado de São Paulo. R. Bras. Zootec., v. 31, n. 3, p. 1150-1156, 2002. Disponível em: $<$ http://www.revista.sbz.org.br/artigo/index.php? artigo $=3207>$

SASA, A.; NONAKA, K. O.; BALIEIRO, J. C. C. et al. Progesterona plasmática de ovelhas submetidas ao efeito-macho e mantidas sob diferentes condições nutricionais. Arq. Bras. Med. Vet. Zootec., v. 63, n. 5, p. 1066-1072, 2011. DOI: 10.1590/S0102-09352011000500004.

STABENFELDT, G. H.; HOLT, J. A.; EWING, L. $L$. Peripheral plasma progesterone levels during the ovine estrous cycle. Endocrinology., v. 85, n. 1, p. 11-15, 1969.. DOI: 10.1210/endo-85-1-11.

VIÑOLES, C.; FORSBERG, M.; BANCHERO, G. et al. Effect of long-term and short-term progestagen treatment on follicular development and pregnancy rate in cyclic ewes. Theriogenology, v.55, n.4, p.993-1004, 2001. Disponível em: <http://www.ncbi.nlm.nih.gov/pubmed/11291921

USTUNER, B. U. GUNAY, U.; NURET, Z. et al. Effects of long and short-term progestagen treatments combined with PMSG on oestrus synchronization and fertility in Awassi ewes during the breeding season. Acta Vet. Brno., v. 76, n. 3, p. 391-397, 2007. DOI: $10.2754 / a v b 200776030391$. 